\title{
Dispositions to Mythmaking Within the Framework of Social Media Activities
}

\author{
Andrey Galukhin \\ Department of History and Philosophy \\ Plekhanov Russian University of Economics \\ Moscow, Russia \\ E-mail: mystolbard@gmail.com
}

\author{
Marina Ivleva \\ Department of History and Philosophy \\ Plekhanov Russian University of Economics \\ Moscow, Russia \\ E-mail: stm602@yandex.ru
}

\author{
Elena Novikova \\ Department of History and Philosophy \\ Plekhanov Russian University of Economics \\ Moscow, Russia \\ E-mail: novikovaelena77@yandex.ru
}

\begin{abstract}
This paper offers a framework for the philosophical understanding of how modern mythmaking is facilitated by social networking, specifically it reveals the preconditions for the proliferation of social and personal myths through social media. The dispositions to mythmaking become manifest in many forms of social networking activities which involve self-presentation and computer-mediated communication and this trend is considered in correlation with the fact that social network services allow their being used as virtual platforms for constructing identities, simulating relationships and thus transcending realistic institutional contexts. The diverse forms of virtual interaction, communication and association in networked communities provide favorable conditions for the initialization of symbolic codes and cultural archetypes which tend to be expressed a quasi-mythological form. This paper considers cognitive trends and identifies factors which strengthen the dispositions to modern mythmaking and enhance susceptibility to the myths distributed through social media. Among the prominent changes in cognitive sphere are the cultivation of computational thinking which accords with technological externalization of the methods of information processing and the actual transformation of cognitive style associated with the emergence of 'mosaic', or 'clip-like thinking'. There is also a correlation between the activation of myth-generative structures of consciousness and the development of a specific language of on-line communication which is characterized by semantic aberrations reflecting the diffusion of the boundaries between the real and virtual worlds. The dispositional properties of social-network relations manifested in the processes of decentralization and self-organization seem to be relevant to the context of mythmaking insofar as mythological creativity can be displayed in building up multidimensional social cyberspace on the basis of bold combinations of realistic and utopian images of sociality.
\end{abstract}

Keywords-social networks; social media; myth; mythmaking; virtual reality; social cyberspace; identity management; clip-like thinking; communication; information technology; multimedia; culture

\section{INTRODUCTION}

Social media networks not only increase the opportunities of communication, interaction and creative activity in cyberspace but also produce extraordinary cultural effects, such as the generation and proliferation of social and personal myths in which new types of identity and forms of social community are encoded. The question of how new social and personal myths originate under the conditions of social networking and what are the mechanisms of their distribution in a shared cyberspace is worth being studied The heuristic value of studying this issue by the means of philosophical analysis becomes evident if we take into account the development of a complex research program oriented at the cross-disciplinary study of social ecology and morphology of cultural phenomena, specifically those phenomena that are characteristic of the information society era. By addressing this issue we reveal an important thematic vector of studying how the increasing use of multimedia technologies for the formation of the information environment and the development of new forms of social communication correlates with the transformation of the cultural matrix of human consciousness in a postmodern situation accompanied by the manifestation of alternative and controversial dispositions to rationalization and mythmaking.

The goal of this propaedeutic research is to expose cultural background of and mental dispositions to mythmaking that become manifest in social media networks and identify the mechanisms for the distribution of mythologems, immanent to the conditions of social-network communication. 


\section{HYPERREALITY: BALANCING ON THE BRINK OF REALITY AND FANTASY}

Being involved in social network communication and cyberspace activities tends to become a rather significant aspect of the reality of human existence. Yet the ontological status of social media presence may seem to be ambiguous because in that case human reality turns out to be restricted to the scope of the possibilities of virtual communication and means of symbolic self-representation, though the mechanisms that would enable to select and structure those opportunities are not always stipulated by the social networks regulation. Social networking and media activities become attractive insofar a person is given the opportunity to determine the measure of the virtualization of his or her presence in the multidimensional cyberspace and choose the means of its symbolization. As E. Efimov points out, "social networks make it possible to balance on the brink of reality and fantasy: a person appreciates social media insofar they offer hyperreal forms in which his life can be represented, but at the same time he partially models his identity" [1]. This balancing may be accompanied by the efforts to bridge the gap between fantasy and reality, yet it makes the difference between virtual and real vague and this can be taken as a spontaneous generator of a sustainable disposition to network myth-making. In fact, this disposition becomes manifest in many cyberspace activities and some of these activities are associated with social networking and virtual communication.

\section{VIRTUAL IDENTITY MANAGEMENT}

Lots of social network users enjoy the opportunities of a virtual "identity management " (VIM): within the framework of VIM the simulative images of the self are multiplied and projected into many forms of virtual presence. Social networks become virtual platforms not only for the replicative assertion of one's identity and creative selfpresentation, but also for the fabrication of many identities, their combination, transformation or simulation. If there are no explicit rules of identity fabrication and if there no preestablished algorithms for determining the extent to which the personal profile of a social network user displays the identity of a real person or represents the artifact of his or her simulative self-projecting, then in the absence of external verifiers and internal constraints all the prerequisites for identity multiplication and identity disaggregation arise. Researchers explain these phenomena in terms of the opportunities offered by social networking sites, they also refer to the general conditions of self-presentation and essential features of social interaction in cyberspace: "While our identities in the real world are socially constructed according to institutional values, cyberspace creates a wider horizontal space that facilitates the personal fabrication of identities. More to the point, whereas real - world identities are generally unitary, in cyberspace identities are frequently multiple. We call this identity disaggregation." [2]. Thus, within the scope of the opportunities of self-presentation and social media communication in cyberspace the channels of multi-level design of our self are opened and the tools of constructing, transforming and camouflaging our identities are employed; there is also a possibility that one can escape from the self through the production of many of its simulative substitutions or discover that his or her identity has been stolen or even deleted [3]. All these devices of virtual life and attributes of online social network activities form the basis for the creation and prolifiration of personal myths. In this case, balancing on the brink of fantasy and reality creates an absolutely schizotypical situation, which post-modernist philosophers like to refer to.

\section{POST-MODERN RE-ENCHANTMENT}

The existential situation of a man living in the world of computer technologies is such that "at the computer interface, the spirit migrates from the body to a world of total representation. Information and images float through the Platonic mind without a grounding in bodily experience. You can lose your humanity at the throw of the dice" [4]. A person may be tempted by a utopia of virtual selfactualization, disembodied existence or even transfiguration, and this utopia becomes a new anthropological myth. Generating forms of virtual reality makes it possible to transcend the boundaries of actual world and travel to other possible worlds. Computer simulations enable to discover and explore alternative universes and this may give birth to a new Platonic myth of transcendent reality. The possibilities of simulating almost any experience and constructing virtual worlds fascinate a person of our time, they promise satisfaction of longing for the transcendent, which has evolved since the very moment when, due to secularization and rationalization, the "disenchantment of the world" (Friedrich Schiller's phrase quoted by M.Weber) had occurred [5]. Predicting the strengthening of ineradicable dispositions to mythmaking correlated to the intensive generation and proliferation of diverse forms of virtual reality, Edward Castronova prophetically claims: "In the long run we are not able to live without myths, . . . and when we see the ongoing migrations of people into lands where magic has finally been credibly (if crudely) rediscovered, we learn how hungry for myth we have become... Perhaps synthetic worlds have begun to offer a new mythology. Perhaps this mythology will eventually be successful, credible, even sublime, so that we will find ourselves in an Age of Wonder" [6]. Thus, the replication of the variable forms of virtual presence provide favorable conditions for the initialization of symbolic codes and mythological archetypes of human consciousness .

\section{VARIABILITY AND MULTIPLICITY}

What are the specific features of mythmaking in the contemporary world? A recent research has shown that there is an evident ideological trend towards the proliferation of modern social mythologies: "there is not just one mythology, but a lot of myths and mythologies" [7] and this situation can be accounted for by the fact that the progressive development of online social networking activities offers some new opportunities for sharing information and distributing ideological simulacra. Contemporary social myths have a limited lifetime, covering, as a rule, not the entire society, but individual groups, and can arise from 
completely different sources. The very infrastructure of social networks facilitates the development of various modes and media formats of network communication and this in turn provides favorable conditions for the simultaneous coexistence and dissemination of various myths and mythologemes which may have 'family resemblance'. A key feature of both the contemporary myth and the classical myth is its' multiplicity and replicability, i.e. the possibility of unlimited proliferation of different versions of the same myth, and coexistence of alternative mythological ideas and subjects. Social networking involves many multimedia activities which increase the opportunities of proliferation of ready-made myths and ideological constructs. The tools for multimedia communication and the terms of association in networked communities not only facilitate infrastructure capabilities of information exchange but also provide technical means of turning social networks into efficient mechanisms for the successive distribution of myths.

\section{TENTATIVE RATIONALITY}

It should be noted that social media users may have completely different potentials of rationality and not every person is capable of exhibiting the ability of being critical about the messages he receives while navigating in the global information space. It is quite evident that welldeveloped abilities of critical thinking are essential to transparent communication and to lots of activities which involve the cases of dealing with a great variety of information content. However, the motivation for using social media can be both rational (it can be explained in terms of "gesellschaft-relations") and irrational (it can be explained in terms of "gemeinschaft-relations") and this fact should be taken into account when determining the measure of the relevance of the critical treatment of the information which flows through computer-mediated communication channels.

On the one hand, the development of Internet and the growth of social media have broken the monopoly on the possession of "asymmetric" information, which is associated with economic influence and bureaucratic power in modern societies: social networking enabled to fully exploit the opportunities of open communication and encouraged almost unrestricted information sharing; the availability of relevant information which can be used in order to acquire knowledge, to accomplish educational tasks and to solve practical problems has increased exponentially. On the other hand, because of the rhizome-like structure of information space and the abstract ways of presenting information on social networking websites it is often the case that the possibilities of tracking the original source of information are reduced and social media users take the risks of being fed with fake news and mistaking myth for reality. If the information is presented without reference to the source and without systematic verification, the possibility of manipulation of consciousness emerges. Social media and networking technologies can be used for the dissemination of ideas, gradually gaining the status of myths or simply attractive because of their quasi-mythological entourage.

\section{COGNITIVE EXTERNALISM}

Moreover, it is quite typical for global social network users to be driven by the technogenic myth which prompts them to cultivate computational thinking; consequently, they may be disposed to transfer the function of selecting and processing information to virtual intelligent machines that function not only in the form of typical search engines but also in the form of selective and distributive mechanisms built into social networking services. Cognitive and functional externalism emerges as an attempt to compensate for the lack of analytical tools, operational resources and cognitive abilities to process big arrays of diverse and discrepant information circulating in the global web, specifically the information which is distributed to people through the use of social media. The archetype of technical "organ projection" originally took shape in the era of scientific and technological revolution; it was enhanced by the proliferation of electronic devices. This is a vital source of strong incentives for believing in the prominent role of technological factors which are assumed to provide a functional substitution of the instruments of thinking (e.g. Artificial Intelligence).

\section{CLIP-LIKE THINKING}

Yet there is another effect of the quasi-existential "abandonment" of a man who has to live in a heterogeneous information environment and who is permanently caught in a web of media technologies employed in the "mass consciousness industry" (the term proposed by M.Mamardashvili). We mean a dramatic cognitive transformation associated with the emergence of the so called 'mosaic thinking' (or "clip-like thinking"). This cognitive style is now becoming widely spread among social media users due to such trends as the increase in the information flow associated with the technological progress and the emergence of mass media, the manifestation of an urgent need for new up-to-date information and the increase in speed of information sharing, the diversification of incoming information and the formation of a heterogeneous media environment (as M.McLuhan claims, "homogeneity is quite incompatible with electronic cuIture" [8], the expansion of the range of activities in which an individual can engage at the same time and the growth of democracy and dialogue at different levels of the social system. The phenomenon of clip-like thinking has become the subject of special cognitive, psychological and phenomenological and cultural research. It is important to study the effects of the operationalization of this style of thinking in the field of education, politics, culture, as well as in everyday practices, including those activities, which involve the use of social networking tools for communication. This cognitive style is characterized by the disposition to be quickly responsive to any impressive informational strike and this disposition correlates with the propensity to switch quickly between the fragments of information and process information on simple schemes and in an accelerated mode; the abstractness of perception of what is presented through the media is also a typical feature of "clip-like thinking". The negative side of this style becomes manifest in the reduction of the ability to 
perceive a long linear sequence - homogeneous information, including that which is encoded in a book text, and the most dramatic effect of this process is the growing inability to bridge semantic gaps or simply to put all the pieces of information together and to form a consistent view of the world. The emergence of mosaic thinking is a good indicator of several trends that are typical for the information society, such as the erosion of the archetypes of textually-centric culture in the mass consciousness. M.McLuhan claimes that "our age translates itself back into the oral and auditory modes because of the electronic pressure of simultaneity" [9] and "we have no more difficulty in understanding the native or non-literate experience, simply because we have recreated it electronically within our own culture " [10] The preference for short sequences of visual symbols over logically related concepts as well as attention deficit displayed in discrete perception of the disconnected units of information are the attributes of 'clip-on thinking'. This cognitive framework is suitable for the persistent replication of contextually heterogeneous ideas that may be paradigmatically associated within the proto-mythological framework induced by media technologies.

\section{Alternative LANGUAGE}

The intensive development of multimedia-technological complexes and their successive implementation in the framework of social media activities provide technical support for the virtualization of social interactions and increase opportunities of simulative substitution of reality in the process of online communication. This in turn stimulates the work of imagination and promotes the dissemination of trans-discursive communicative practices thus strengthening the need for a new language, alternative to that which is used in the framework of classical textualization. Dispositions and means for rooting and unfolding a variety of mythologems become manifest in the structure of "language games" played by social media users. Thus, describing the way the myth exists, Roland Barthes claims that "Myth is a type of speech" [11]. "Speech of this kind is a message. It is therefore by no means confined to oral speech. It can consist of modes of writing or of representations; not only written discourse, but also photography, cinema, reporting, sport, shows, publicity, all these can serve as a support to mythical speech" [12]. Multimedia technologies enable to operate with different types of information content and expand the range of technical means of communication; most notably they help to establish some new formats of information coding which involve the use of both standard means of symbolization and non-standard techniques oriented at the forms of semiosis that fit in with the design of virtual "language games". Diffusion of the boundaries of the real and virtual worlds is often reflected by the semantic duality and syntactic polymorphism of the language of social media users. The most important thing is that both canonical and non-canonical, e.g., textual and visual, sound, kinetic and even kinesthetic codes can be used for self-presentation and on-line communication. Such a combination of traditional and extra-ordinary ways in which meanings can be conveyed facilitates the proliferation of myths through social media. That is because "mythical speech" can take an adequate symbolic form and can be delivered in a variety of alternative ways. Due to the shifting forms and changing degrees of virtualization of individual presence and interactive relations, networking activities can take the form of "post-symbolic communication". Thus, addressing the issues of on-line communication and revealing the conditions of building semantic continuums in a set of virtual worlds, M.Heim notes that "communication can go beyond verbal or body language to take on magical, alchemical properties. A virtual-world maker might conjure up hitherto unheardof mixtures of sight, sound, and motion. Consciously constructed outside the grammar and syntax of language, these semaphores defy the traditional logic of verbal and visual information" [13]. Social media incorporate the mechanisms for the dissemination and circulation of myths and this can be partly accounted for by the fact that linguistic and extra-linguistic forms of social-network communication can be easily transformed into the forms of virtual public life that share one and the same feature - living in the multidimensional symbolical space of the myth.

\section{SOCIAL SYNERGETIC EFFECTS IN RHIZOMORPHOUS CYBERSPACE}

Social media development expands the sphere of infrastructural preconditions for the multiplication and proliferation of social and personal myths: distributive capacities of social networks are facilitated by their dispositional properties displayed in the processes of decentralization and self-organization. It is commonly known that the term "social networks" was proposed by J.A.Barnes [14]. Originally this term referred to a set of informal extra-institutional social relations characterized by the absence of a "coordinated organization" based on cultural prescriptions or induced by power. Social networks are characterizes by the tendency toward decentralization and the capacity of self-organization and these dispositional properties correlate well with the structural characteristics of the global information space: "The new information networks resemble the modern megalopolis, often described as a concrete jungle (New York) or a sprawl (Los Angeles). A maze of activities and hidden byways snakes around with no apparent center. Architecturally, the network sprawl suggests the absence of a philosophical or religious absolute" [15]. In this context it would be appropriate to draw a cultural and historical analogy with ancient polytheistic systems: there was a diversity of local cults in ancient Greece each of which incorporated or prioritized a particular system of mythologems. Ancient cults and myths of the land multiplied, overlapped and proliferated and that was quite natural in a particular cultural situation which was marked by the absence of the universal frame of reference associated with the absolute religious authority or the ultimate philosophical truth. Indeed, that was the reverse side of Archaic polytheism. In our days social network relations obtain a new ontological status - they have developed as a substantial part of virtual reality and their dispositional properties such as decentralization and self-organization are amplified by the polymorphic structure of a shared information space which is organized as the rhizome of interrelated and all-pervading hyperlinks. Rhizomorphic 
complexity of information space shared by Internet users meets the standards established in accordance with a postmodernist strategy of the deconstruction of monological systems of meanings. Polycentrism of meanings together with the irreducible heterogeny of information and selforganizational capacity of social networks provide favorable conditions for the formation of a specific mindset of social media users: their multiplying quazi-mythological views turn out to be ideological analogues of ancient cults, which coexisted without any prospect of being integrated into the universal system of values and beliefs.

\section{SUB-CUltural CREATIVITY IN VIRTUAL COMMUNITIES}

The development of virtual social communities is a rather notable effect of social networking. The diversity of forms of communication and association between social media users provide the best verification of the idea of cyberspace as an open and dynamic social environment. Virtual communities can be created as user associations based on their shared real interests and relationships. Social researchers are right when they claim that "in terms of their major characteristics (interactions, boundaries and social mores) and with respect to the drivers of their development the associations of network users are similar to the typical objects of a traditional sociological description" [16]. Virtual communities are also an interesting object of social and cultural research. Those communities often have a rather specific sub-cultural system which incorporates virtual artifacts, forms of self-presentation, norms of communication and means of articulation of various interests of networked participants; virtual communities' subcultures are reproduced through a set of established traditions and rituals, and myths and stories may be of crucial importance in this context they are designed to transfer symbolic codes and values which serve as the basis for self-identification and group integration. Within the framework of realistic social analysis it is quite typical to explain a rationale for interactive communication between the members of social media communities in terms of pragmatic intentions such as the intention to acquire or increase social capital measured in such variables as status, reputation, available information, influence, power and the scope of social relations [17]. And that is apart from satisfying fundamental communication needs and demands for self-expression, recreation, sharing of experience, education, attaining solidarity etc. But realistic account of socially interactive practices is likely to degenerate into substantial reductionism when it loses the sight of those characteristics of virtual communities which are conditioned by constructive attitude of their members to the intrinsic forms of virtual reality enabling networked communicators to use media resources and technologies and employ their own creative potential for the development of social cyberspace. The fact is that virtual self-presentation, association and communication through social media can be thought of alternatively: it may be the case of the extensive replication of the real forms of practical interaction or everyday communication; yet, social media activities may converge in a complex social role game which involves symbolic exchange, confrontation or cooperation, communication or strategic action as well as the establishment of various relationships and the formation of various groups representing structural and dynamic features of a virtually developing society. It seems that social media users are justified in holding the view that social networking and community building in virtual reality allows for the deconstruction of the actual paradigms of social organization and opens the way for building up social relationships on a new ideological platform which in fact may be analogues to the "original position" conceptualized by John Rawls in his theory of justice. This view can be easily transformed into a set of myths of social innovation distributed through social media [18].

\section{VIRTUAL PlatFormS FOR SOCIAL ENGINEERING}

We can conceive of on-line communication through social media as a form activity which provides operationalization of individual and social presence in the virtual world. Yet, there may be a great deal of divergence with social reality: "the virtual world creates spaces where fame, prestige, esteem, influence and even wealth are conferred according to an entirely different system of values. Virtual environments create level playing fields where traditional attributes that confer status are regarded not only as unjust and inefficient, but also irrelevant" [19]. The "democratization of status" in social cyberspace implies that the concept of social capital has been reconsidered and modified in accordance with a new understanding of the nature and quality of relationships between the members of virtual network communities. A new framework of interpersonal and social relationships is established in the process of multimedia based interaction and social network communication which involves sharing attitudes not only to the real world but also to a set of virtual worlds in which the interplay of alternative values and meanings of sociality takes place. When there is asymmetry between these two attitudes the concept of social capital turns out to be informed with hyper-symbolic content and its' meaning is likely to acquire a mythical shade. This is the case when the modes and forms of representation of an individual as a member of virtual network community affect and transform the perception of his or her personality or position in a system of real social relations thus inducing the disposition to evaluate personal achievement and social status through the system of simulative axiological constructs.

\section{CONCLUSION}

Social media offer great opportunities for synergetic creativity which can be fully displayed in producing heuristic simulative images of social reality: on the one hand, those artifacts of virtual social constructing gives expression to urgent demands for social reforms and progressive innovations in particular areas of society and their function may be identical with that of utopia which can be used as an instrument of social criticism; on the other hand, among the ideological products of social media activities there is a variety of symbolic constructs of social world that have some mythological content, social constructs are myth-bearers and it is due to this fact that social media are transformed into the 
mechanisms of the successive distribution of social myths. Insofar social media serve as virtual platforms for the formation of various cyber societies the question arises about the limits, structure and intention of social constructing, which is the question of whether this activity inevitably leads to the facilitation of the dispositions to unrealistic mythmaking or has some realistic goal and heuristic function. Our answer is: synergetic creative activity in social cyberspace may have positive heuristic value and realistic orientation inasmuch as virtual identity management and social constructing fulfill the functions of thought experiment which enables to examine possible social worlds, articulate implicit social trends and find new ways of the real social and cultural communities' development.

\section{REFERENCES}

[1] Efimov, E.G. 2015, Online social networking (research methodology and practice). Volgograd: Volgograd State Technical University. P.7

[2] Fraser, Matthew and Soumitra, Dutta, 2008, Throwing Sheep in the Boardroom. How Online Social Networking Will Transform Your Life, Work and World. John Wiley \& Sons Ltd. P.20

[3] Fraser, Matthew and Soumitra, Dutta, 2008, Throwing Sheep in the Boardroom. How Online Social Networking Will Transform Your Life, Work and World. John Wiley \& Sons Ltd. P.21

[4] Heim, M. 1991, The Metaphysics of Virtual Reality. Oxford: Oxford University Press. p.101

[5] Weber, Max. 1946, Religious Rejections of the World and Their Directions. In: Gerth H.H. and Mills Wright C. (eds.) From Max Weber: Essays in Sociology, pp. 323 - 359, New York: Oxford University Press. P.350

[6] Castronova, Edward, 2006, Synthetic Worlds: The business and culture of online games. Chicago: Chicago University Press. p.276

[7] McLuhan, Herbert Marshall, 1962, The Gutenberg Galaxy: The Making of Typographic Man. Toronto: University of Toronto Press.

[8] McLuhan, Herbert Marshall, 1962, The Gutenberg Galaxy: The Making of Typographic Man. Toronto: University of Toronto Press.P.135

[9] McLuhan, Herbert Marshall, 1962, The Gutenberg Galaxy: The Making of Typographic Man. Toronto: University of Toronto Press.P.72

[10] McLuhan, Herbert Marshall, 1962, The Gutenberg Galaxy: The Making of Typographic Man. Toronto: University of Toronto Press.P. 46

[11] Barthes, Roland, 1991, Mythologies. Selected and translated from the French by Annette Lavers. New York : Noonday Press. P.107

[12] Barthes, Roland, 1991, Mythologies. Selected and translated from the French by Annette Lavers. New York : Noonday Press. P.108

[13] McLuhan, Herbert Marshall, 1962, The Gutenberg Galaxy: The Making of Typographic Man. Toronto: University of Toronto Press. P. 116

[14] Barnes, J.A. 1954, Class and committees in a Norwegian island parish, Human Relations, vol. 7, pp. 39-58. P.43

[15] McLuhan, Herbert Marshall, 1962, The Gutenberg Galaxy: The Making of Typographic Man. Toronto: University of Toronto Press. P. 104

[16] Efimov, E.G. 2015, Online social networking (research methodology and practice). Volgograd: Volgograd State Technical University. P.30

[17] Ellison, N.B., Lampe, C., Steinfield, Ch., and Vitak J. 2011, With a Little Help From My Friends How Social Network Sites Affect Social Capital Processes In: Zizi A. Papacharissi (ed.) A networked self : identity, community and culture on social network sites, pp. 124 - 145 , New York: Routledge.
[18] Toporkov, A.L., 2011, Myths and Mythology in the XX century: traditions and perceptions. In: Proceedings of the research seminar "Folklore and Post-Folklore: Structure, Typology and Semiotics". Centre for Typological and Semiotic Folklore Studies, Russian State University for the Humanities URL http://www.ruthenia.ru/folklore/toporkov1.htm (accessed May 24, 2018).

[19] Fraser, Matthew and Soumitra, Dutta, 2008, Throwing Sheep in the Boardroom. How Online Social Networking Will Transform Your Life, Work and World. John Wiley \& Sons Ltd. P.22. 